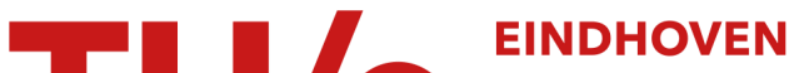 \\ UNIVERSITY OF \\ TECHNOLOGY
}

\section{Current-in-plane magnetoresistance : a new approach to boundary conditions}

\section{Citation for published version (APA):}

Litvinov, V. I., Dugaev, V. K., Willekens, M. M. H., \& Swagten, H. J. M. (1997). Current-in-plane magnetoresistance : a new approach to boundary conditions. Physical Review B: Condensed Matter, 55(13), 8374-. https://doi.org/10.1103/PhysRevB.55.8374

DOI:

10.1103/PhysRevB.55.8374

Document status and date:

Published: 01/01/1997

\section{Document Version:}

Publisher's PDF, also known as Version of Record (includes final page, issue and volume numbers)

\section{Please check the document version of this publication:}

- A submitted manuscript is the version of the article upon submission and before peer-review. There can be important differences between the submitted version and the official published version of record. People interested in the research are advised to contact the author for the final version of the publication, or visit the $\mathrm{DOI}$ to the publisher's website.

- The final author version and the galley proof are versions of the publication after peer review.

- The final published version features the final layout of the paper including the volume, issue and page numbers.

Link to publication

\section{General rights}

Copyright and moral rights for the publications made accessible in the public portal are retained by the authors and/or other copyright owners and it is a condition of accessing publications that users recognise and abide by the legal requirements associated with these rights.

- Users may download and print one copy of any publication from the public portal for the purpose of private study or research.

- You may not further distribute the material or use it for any profit-making activity or commercial gain

- You may freely distribute the URL identifying the publication in the public portal.

If the publication is distributed under the terms of Article 25fa of the Dutch Copyright Act, indicated by the "Taverne" license above, please follow below link for the End User Agreement:

www.tue.nl/taverne

Take down policy

If you believe that this document breaches copyright please contact us at:

openaccess@tue.nl

providing details and we will investigate your claim. 


\title{
Current-in-plane magnetoresistance: An approach to boundary conditions
}

\author{
V. I. Litvinov and V. K. Dugaev \\ Institute of Material Science Problems, Ukranian Academy of Sciences, 5 Wilde Street, 274001, Chernovtsy, Ukraine \\ M. M. H. Willekens and H. J. M. Swagten \\ Department of Physics, Eindhoven University of Technology, P.O. Box 513, 5600 MB Eindhoven, The Netherlands
}

(Received 9 July 1996)

\begin{abstract}
The giant magnetoresistance effect is calculated for metallic magnetic superlattices with current-in-plane geometry by solving the semiclassical Boltzmann transport equation. Two types of interface roughness will be taken into account, geometrical interface roughness and scattering at defects, impurities, etc. located at the interface. Within this approach, geometrical interface roughness with a finite correlation length is considered. It is shown that geometrical interface roughness, without any other form of spin-dependent scattering, can result in a considerable magnetoresistance. [S0163-1829(97)00213-0]
\end{abstract}

\section{INTRODUCTION}

The giant magnetoresistance (GMR) in metallic multilayers like $\mathrm{Co} / \mathrm{Cu}$ and $\mathrm{Fe} / \mathrm{Cr}$ is a well-known phenomenon and generally it is accepted that the GMR effect finds its origin in different scattering rates for spin-up and spin-down electrons. ${ }^{1}$ The relative contribution to the scattering rates from bulk and interfacial scattering processes is considered as an important problem in recent investigations. To describe interfacial current transmission several theoretical approaches have been developed and one could divide them in two main groups, one of which employs the semiclassical Boltzmann approach ${ }^{2,3}$ (BA) and another using a quantum approach $^{4-6}$ based on the Kubo formalism. When the size quantization across the layers can be neglected, any of the approaches mentioned above can be used to describe the transport properties in a proper way. ${ }^{7}$

A major goal of any theoretical model for describing the GMR effect is to take into account in a most consistent way the interfacial scattering or nonideality of the interfaces, which is generally separated in two different contributions, namely geometrical roughness and electron scattering on defects and impurities located at the interfaces. In the BA this problem mainly corresponds to the formulation of proper boundary conditions for the electron distribution functions. These boundary conditions are supposed to include the properties of the interfaces.

An attempt to describe the nonideality of the interfaces in magnetic multilayer systems within the BA was made by Camley and Barnas. ${ }^{2}$ They modeled the interfaces by two purely phenomenological spin-dependent parameters, one for coherent transmission and one for diffusive scattering. However, whether or not an electron experiences the interface as a specular plane depends crucially on its momentum and thus on the angle of incidence on the interface which was not included in the interface parameters. Thus, with the parameters in the Camley-Barnas model it is impossible to discern between the characteristics of the incident electrons and the properties of the interfaces.

Therefore, Hood and Falicov ${ }^{3}$ made a more realistic approach. They introduced different potentials for majority and minority spins in the magnetic layers. Then coefficients for coherent transmission and specular reflection can be quantum mechanically calculated by matching free-electron-like plane-wave functions and their derivatives at each interface. Since in this microscopical approach the boundary conditions for the distribution functions depend on the electron velocity, they automatically also depend on the angle of incidence. Unfortunately their model still contains one purely phenomenological spin-dependent parameter that denotes the degree of potential scattering (the rest being scattered diffuse) and decreases with the concentration of defects and impurities located at the interfaces. The calculated GMR depends crucially on the value of this parameter.

As far as geometrical roughness is concerned, Hood, Falicov, and Penn ${ }^{3}$ (HFP) have only considered one special case when the in-plane roughness correlation length $L$ tends to zero. This limit however does not allow one to consider the processes of the diffusive electron scattering in a proper way since physically $L$ is limited to the atomic size.

Recently Barnas ${ }^{8}$ reported on a quantum-mechanical approach where the geometrical interface roughness is determined by two statistical parameters, the root mean square $\eta$ of the deviations from the perfectly flat interface and the in-plane correlation length $L$. Here, the scattering on the geometrical roughness is treated equivalent to the scattering on impurities and defects at the interface, i.e., by potential scattering.

In this paper we will describe a semiclassical model based on the BA. In fact it is an extension of the model used by HFP. $^{3}$ As in the model of Barnas ${ }^{8}$ we will define the interface roughness with the statistical parameters $\eta$ and $L$. We will not assume however that the electrons experience the curvature of the interface as a special external potential field, but we will calculate the boundary conditions by matching the electron wave functions on the actual position of the nonideal interface. All potential scattering agents at the interface, like defects and impurities, but also the potential scattering on the bumps, are contained in a random field $W(\boldsymbol{\rho})$. The method used for the description of scattering at the interfaces has been proposed by Falkovsky ${ }^{9}$ and has recently ${ }^{10}$ been applied to study impurity scattering at a flat 


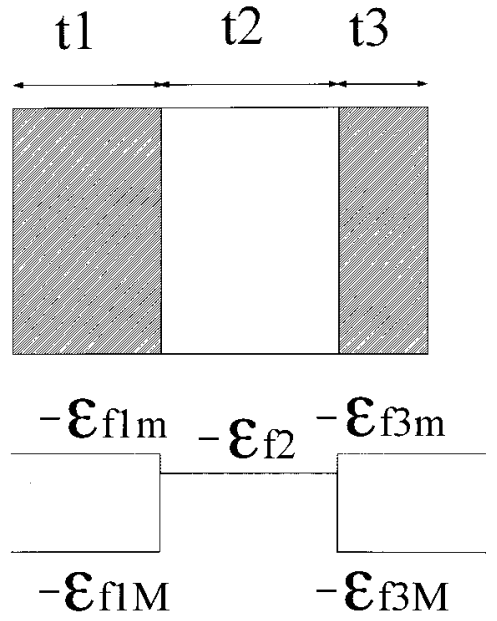

FIG. 1. Schematic diagram of the potential landscape within the surperlattice.

interface between two simple nonmagnetic metals. We have extended this method for magnetic superlattices with currentin-plane geometry. Our model does not contain any phenomenological parameter and the main advantage is that it enables one to examine the mechanism of effective scattering on roughness which arises from pure geometrical properties of the interface.

\section{FORMULATION OF THE MODEL}

Consider a trilayer consisting of two ferromagnetic layers of thickness $t_{1}$ and $t_{3}$ separated by a nonmagnetic spacer of thickness $t_{2}$. All metals are regarded as free-electron like and the energy diagram ${ }^{3}$ of the system under consideration is shown Fig. 1. The Fermi energies $\varepsilon_{f 1,3}$ depend on the spin direction. We assume spin-up to be parallel to the magnetization in layer 1 . In the parallel $(\mathrm{P})$ configuration the magnetizations of layers 1 and 3 will point in the same direction, in the antiparallel (AP) configuration the magnetization direction of layer 3 will be opposite to that of layer 1 . Since we want to consider a superlattice the two outer surfaces of the trilayer are regarded as perfectly flat and they are assumed to reflect all incident electrons specularly. Only the interfaces $1 / 2$ and $2 / 3$ between metals 1,2 and 2,3, respectively, are regarded as nonideal. There are two sorts of nonideality that we will take into consideration: (a) scatterers distributed over the plane and (b) geometrical roughness.

For a single flat interface between media 1 and 2 the Schrödinger equation for an electron's wave function $\Psi(\mathbf{r})$ has the form ${ }^{10}$

$$
\begin{aligned}
& -\frac{\hbar^{2}}{2 m} \nabla[1-\Theta(z)] \nabla \Psi_{1}-\frac{\hbar^{2}}{2 m} \nabla \Theta(z) \nabla \Psi_{2}+W(\boldsymbol{\rho}, z) \Psi \\
& \quad=\varepsilon_{1} \Psi[1-\Theta(z)]+\varepsilon_{2} \Psi \Theta(z),
\end{aligned}
$$

in which $\boldsymbol{\rho}=(x, y)$ is the in-plane coordinate, $\Theta(z)$ is the unit step function, and $W(\boldsymbol{\rho}, z)$ is a random scattering potential caused by impurities and other possible potential scattering agents. We consider only the simplest case $W(\boldsymbol{\rho}, z)$ $=\delta(z) W(\boldsymbol{\rho})$ and assume that the distribution of the random field $W(\boldsymbol{\rho})$ is Gaussian with a $\delta$-like correlator that corresponds to short-range scattering centers:

$$
\gamma^{\prime}(\rho)=\langle W(0) W(\boldsymbol{\rho})\rangle=\gamma^{\prime} \delta(\boldsymbol{\rho}) \quad \text { with } \quad \gamma^{\prime}=N_{i} W_{0}^{2}
$$

where $N_{i}$ is the planar concentration of scattering centers, $W_{0}$ is the Fourier transform of the potential, and \langle\rangle means averaging over all realizations of $W(\boldsymbol{\rho})$.

Another source of nonideality of the interface is geometrical roughness which is described by random deviations from a perfectly flat interface $\zeta(\boldsymbol{\rho})$. We assume a Gaussian distribution of $\zeta$ with spatial correlation taken into account in the following way:

$$
\gamma(\boldsymbol{\rho})=\langle\zeta(0) \zeta(\boldsymbol{\rho})\rangle=\eta^{2} \exp \left(-\frac{\rho^{2}}{L^{2}}\right),
$$

where $\eta=\sqrt{\left\langle\zeta^{2}(\boldsymbol{\rho})\right\rangle}$ is the root-mean-square value of the deviations. The two-dimensional Fourier transform has the form

$$
\gamma(\mathbf{q})=\frac{\eta^{2} L^{2}}{2} \exp \left(-\frac{q^{2} L^{2}}{4}\right),
$$

where $\hbar q$ is the in-plane momentum, $L$ is the correlation length, which has the physical meaning of the average size of smooth parts of the interface, and \langle\rangle means averaging over all realizations of $\zeta$.

\section{CONDUCTIVITY}

The general solution to the Boltzmann equation for media with a single boundary at $z=0$ parallel to the $x-y$ plane with an applied electrical field $E$ in the $x$ direction has the form

$$
f^{ \pm}=f_{0}+\frac{e \tau E}{m} \frac{\partial f_{0}}{\partial v_{x}}\left[1+F^{ \pm} \exp \left(\mp \frac{z}{\tau\left|v_{z}\right|}\right)\right],
$$

where $f_{0}$ is the Fermi-Dirac equilibrium distribution function, $\tau$ denotes the bulk relaxation time, $v_{z}$ is the $z$ component of the electron velocity, coefficients $F^{ \pm}$are determined from the boundary conditions and \pm corresponds to a positive or negative velocity with respect to the $z$ direction.

We start from the equation for in-plane conductivity for the $\mathrm{P}$ configuration. For the sake of simplicity spin-flip scattering processes have not been taken into account. Thus

$$
\begin{aligned}
\sigma_{\uparrow \uparrow}= & \frac{1}{E t_{\text {tot }}}\left\{\sum _ { \sigma } \left[\int_{0}^{t_{1}} J_{1 \sigma}(z) d z+\int_{t_{1}}^{t_{1}+t_{2}} J_{2 \sigma}(z) d z\right.\right. \\
& \left.\left.+\int_{t_{1}+t_{2}}^{t} J_{3 \sigma}(z) d z\right]\right\},
\end{aligned}
$$

where $J_{1,2,3}$ are current densities in layers 1,2 , and 3, respectively, which depend on the relative orientation of the magnetic moments in layers 1 and 3 and $t_{\text {tot }}=t_{1}+t_{2}+t_{3}$.

In order to calculate the conductivity in superlattices we use distribution functions as given by Eq. (5) adjusted to include interfaces at $z \neq 0$. We assume that the outer boundaries of our trilayer, at $z=0$ and $z=t_{\text {tot }}$, act as a mirror to all incident electrons (completely specular reflection), which means that in fact we study an infinite superlattice with a 
repetition unit of $2 t_{1} / t_{2} / 2 t_{3} / t_{2}$. This assumption results in the following boundary conditions for the outer boundaries:

$$
f_{1 \sigma}^{+}=f_{1 \sigma}^{-} \quad \text { at } \quad z=0 \quad \text { and } \quad f_{3 \sigma}^{+}=f_{3 \sigma}^{-} \quad \text { at } \quad z=t_{\text {tot }} .
$$

Appropriate boundary conditions for the inner interfaces will be derived in the next section. Calculating current densities with help of Eqs. (6) and (7) we arrive at the equation

$$
\sigma_{\uparrow \uparrow}=\sigma^{0}+\sigma_{\uparrow \uparrow}^{1}+\sigma_{\uparrow \uparrow}^{2}+\sigma_{\uparrow \uparrow}^{3},
$$

where $\sigma^{0}$ is the bulk conductivity. The deviations from the bulk behavior due to interfaces are represented by $\sigma_{\uparrow \uparrow}^{1}$, $\sigma_{\uparrow \uparrow}^{2}$ and $\sigma_{\uparrow \uparrow}^{3}$ where

$$
\begin{aligned}
& \sigma_{\uparrow \uparrow}^{1}= \frac{e^{2}}{2 \pi^{2} \hbar^{3} t_{\text {tot }}} \sum \varepsilon_{f 1 \sigma}^{2} \tau_{1 \sigma}^{2} \int_{0}^{1} F_{1 \sigma}^{-}\left(x_{1 \sigma}\right) \\
& \times\left[1-\exp \left(\frac{-2 t_{1}}{\lambda_{1 \sigma} x_{1 \sigma}}\right)\right]\left(1-x_{1 \sigma}^{2}\right) x_{1 \sigma} d x_{1 \sigma}, \\
& \sigma_{\uparrow \uparrow}^{2}= \frac{e^{2}}{\pi^{2} \hbar^{3} t_{\text {tot }}} \varepsilon_{f 2}^{2} \tau_{2}^{2} \int_{0}^{1}\left[F_{2}^{+}\left(x_{2}\right)+F_{2}^{-}\left(x_{2}\right)\right]\left[\exp \left(\frac{-t_{2}}{\lambda_{2} x_{2}}\right)-1\right] \\
& \times\left(1-x_{2}^{2}\right) x_{2} d x_{2}, \\
& \sigma_{\uparrow \uparrow}^{3}=\frac{e^{2}}{2 \pi^{2} \hbar^{3} t_{\text {tot }}} \sum_{\sigma} \varepsilon_{f 3 \sigma}^{2} \tau_{3 \sigma}^{2} \int_{0}^{1} F_{3 \sigma}^{+}\left(x_{3 \sigma}\right) \\
& \quad \times\left[1-\exp \left(\frac{-2 t_{3}}{\lambda_{3 \sigma^{2}} x_{3 \sigma}}\right)\right]\left(1-x_{3 \sigma}^{2}\right) x_{3 \sigma} d x_{3 \sigma},
\end{aligned}
$$

where $x_{i \sigma} \equiv v_{i \sigma} / v_{f i \sigma}$ with $v_{i \sigma}=(1 / m) \sqrt{2 m \varepsilon_{f i \sigma}-\hbar^{2} k^{2}}$, the $z$ component of the electron velocity on the Fermi surface in layer $i, \hbar k$ is the modulus of the in-plane momentum, $v_{f i}$ is the Fermi velocity in layer $i$, and $\lambda_{i \sigma}$ is the bulk mean free path in layer $i$.

Similar expressions can be obtained for the AP configuration with correspondent coefficients $F_{i \sigma}^{ \pm}$. For both configurations we can identify Fermi energies and bulk relaxation times of electrons with their majority $(M)$ and minority $(m)$ values:

$$
\begin{array}{ll}
\text { (P): } \quad \tau_{1 \uparrow \downarrow}=\tau_{1 M m} ; \tau_{3 \uparrow \downarrow}=\tau_{3 M m}, \\
(\mathrm{AP}): \quad \tau_{1 \uparrow \downarrow}=\tau_{1 M m} ; \tau_{3 \uparrow \downarrow}=\tau_{3 m M} .
\end{array}
$$

To perform the final calculation in Eq. (9) one should find the coefficients $F_{i \sigma}^{ \pm}$for both (P) and (AP) configurations making use of the boundary conditions for the distribution functions. In the next section we will derive the boundary conditions from a microscopical consideration.

\section{BOUNDARY CONDITIONS FOR DISTRIBUTION FUNCTIONS}

First we consider the wave functions for a single interface ${ }^{10}$ which are wave packets on the Fermi surface in metals 1 and 2:

$$
\begin{aligned}
\Psi_{1,2}(\varepsilon, z, \boldsymbol{\rho})= & \int d \mathbf{k} \frac{\exp (i \mathbf{k} \cdot \boldsymbol{\rho})}{v_{1,2 k}}\left[a_{1,2 k}^{<} \exp \left(-i k_{1,2 z} z\right)\right. \\
& \left.+a_{1,2 k}^{>} \exp \left(i k_{1,2 z} z\right)\right],
\end{aligned}
$$

in which the coefficients $a^{<}$and $a^{>}$correspond to $k_{z}=-k_{1,2 z}$ and $k_{z}=+k_{1,2 z}$, respectively, $k_{1,2 z}=m_{1,2} v_{1,2} / \hbar$, $v_{i k \sigma}$ is the $z$ component of the electron velocity on the Fermi surface in layer $i$, and $\hbar k$ is the modulus of the in-plane momentum. The electron distribution functions are related to the coefficients $a^{<}, a^{>}$via $^{9}$

$$
\left|a_{1 k}^{<}\right|^{2}=v_{1 k} f_{1}^{-} ;\left|a_{1 k}^{>}\right|^{2}=v_{1 k} f_{1}^{+} ;\left|a_{2 k}^{<}\right|^{2}=v_{2 k} f_{2}^{-}
$$

and

$$
\left|a_{2 k}^{>}\right|^{2}=v_{2 k} f_{2}^{+} .
$$

From the Schrödinger equation [Eq. (1)] one can obtain the matching conditions for quantum-mechanical currents at the interface. ${ }^{10}$ The matching of wave functions and currents at a nonideal, curved interface between metals 1 and 2 leads to the following equations:

$$
\Psi \equiv \Psi_{1}=\Psi_{2} \quad \text { at } \quad z=\zeta(\boldsymbol{\rho})
$$

and

$$
\left.\frac{\hbar^{2}}{2 m_{1}} \frac{d \Psi_{1}}{d z}\right|_{z=\zeta-\delta}-\left.\frac{\hbar^{2}}{2 m_{2}} \frac{d \Psi_{2}}{d z}\right|_{z=\zeta+\delta}+W(\boldsymbol{\rho}) \Psi=0, \quad \delta \rightarrow 0 .
$$

From Eqs. (13) we first obtain ${ }^{9}$ relations between coefficients $a^{<}, a^{>}$and, thereafter, with help of Eq. (12), the boundary conditions for electron distribution functions at the interface. After averaging these relations over all the realizations of random variables $W(\boldsymbol{\rho})$ and $\zeta(\boldsymbol{\rho})$ the boundary conditions take the form (assuming $m_{1}=m_{2} \equiv m$ ):

$$
\begin{aligned}
f_{1 k \sigma}^{-}= & R_{12 \sigma} f_{1 k \sigma}^{+}+T_{12 \sigma} f_{2 k \sigma}^{-}+\int d^{2} q\left[A_{12}(\mathbf{k}, \mathbf{q}) f_{1(k+q) \sigma}^{+}\right. \\
& \left.+B_{12}(\mathbf{k}, \mathbf{q}) f_{2(k+q) \sigma}^{-}\right], \\
f_{2 k \sigma}^{+}= & R_{21 \sigma} f_{2 k \sigma}^{-}+T_{21 \sigma} f_{1 k \sigma}^{+}+\int d^{2} q\left[A_{21}(\mathbf{k}, \mathbf{q}) f_{1(k+q) \sigma}^{+}\right. \\
& \left.+B_{21}(\mathbf{k}, \mathbf{q}) f_{2(k+q) \sigma}^{-}\right],
\end{aligned}
$$

where

$$
A_{12}(\mathbf{k}, \mathbf{q})=v_{1 k \sigma} v_{1(k+q) \sigma} Q_{12}, \quad B_{12}(\mathbf{k}, \mathbf{q})=v_{1 k \sigma} v_{2(k+q)} Q_{12}
$$

and

$$
\begin{aligned}
& A_{21}(\mathbf{k}, \mathbf{q})=v_{2 k} v_{1(k+q) \sigma} Q_{12}, \quad B_{21}(\mathbf{k}, \mathbf{q})=v_{2 k} v_{2(k+q)} Q_{12} \\
& Q_{12}= \frac{2}{\pi \hbar^{2}\left(v_{1 k \sigma}+v_{2 k}\right)^{2}}\left[m^{2}\left(v_{1(k+q) \sigma}-v_{2(k+q)}\right)^{2} \gamma_{12}(\mathbf{q})\right. \\
&\left.+\frac{4 \gamma_{12}^{\prime}(\mathbf{q})}{\left(v_{1(k+q) \sigma}+v_{2(k+q)}\right)^{2}}\right]
\end{aligned}
$$




$$
\begin{gathered}
R_{12}=\widetilde{R}_{12}+\Delta R_{12}, \quad T_{12}=\widetilde{T}_{12}+\Delta T_{12}, \\
\widetilde{R}_{12}=R_{12}^{0}\left(1-\frac{4 m^{2} \eta^{2} v_{1 k \sigma} v_{2 k}}{\hbar^{2}}\right)
\end{gathered}
$$

with

$$
R_{12}^{0}=\left(\frac{v_{1 k \sigma}-v_{2 k}}{v_{1 k \sigma}+v_{2 k}}\right)^{2}
$$

and

$$
\widetilde{T}_{12}=T_{12}^{0}\left(1+\frac{m^{2} \eta^{2}\left(v_{1 k \sigma}-v_{2 k}\right)^{2}}{\hbar^{2}}\right)
$$

with

$$
T_{12}^{0}=\frac{4 v_{1 k \sigma} v_{2 k}}{\left(v_{1 k \sigma}+v_{2 k}\right)^{2}} .
$$

Corrections caused by effective interface scattering processes have the form

$$
\begin{aligned}
\Delta T_{12}= & \Delta T_{21}=T_{12}^{0}\left\{\frac { m ^ { 2 } ( v _ { 1 k \sigma } - v _ { 2 k } ) } { \pi \hbar ^ { 2 } } \int \gamma ( \mathbf { q } ) \left(v_{2(k+q)}\right.\right. \\
& \left.\left.-v_{1(k+q) \sigma}\right) d^{2} q-\frac{4}{\pi \hbar^{2}\left(v_{1 k \sigma}+v_{2 k}\right)} \int \frac{\gamma^{\prime}(\mathbf{q}) d^{2} q}{v_{1 k \sigma}+v_{2 k}}\right\}
\end{aligned}
$$

and

$$
\begin{aligned}
\Delta R_{12}= & R_{12}^{0}\left\{\frac{2 v_{1 k \sigma} m^{2}}{\pi \hbar^{2}} \int \gamma(\mathbf{q})\left(v_{2(k+q)}-v_{1(k+q) \sigma}\right) d^{2} q\right. \\
& \left.-\frac{8 v_{1 k \sigma}}{\pi \hbar^{2}\left(v_{f 1 \sigma}^{2}-v_{f 2}^{2}\right)} \int \frac{\gamma^{\prime}(\mathbf{q}) d^{2} q}{v_{1(k+q) \sigma}+v_{2(k+q)}}\right\} .
\end{aligned}
$$

Similar boundary equations can be obtained for the interface between layers 2 and 3 where $\gamma(\mathbf{q})$ and $\gamma^{\prime}(\mathbf{q})$, describing the properties of the second interface, may be different. Ranges of integrations in Eqs. (17) and (18) are determined by the simultaneous existence of $z$ components of the Fermi velocities in adjacent layers. Conditions (16) have been obtained with second-order accuracy in $W(\boldsymbol{\rho})$ and $\zeta(\boldsymbol{\rho})$ and no mixed terms like $W(\boldsymbol{\rho}) \zeta(\boldsymbol{\rho})$ were taken into account. This means that we neglect the influence of roughness on impurity scattering and assume that these two factors act independently. The boundary conditions of Eq. (14) are integral equations which were solved in first-order approximation in scattering and roughness correlators, Eqs. (2) and (4). It appears that the integral terms in Eq. (14), when substituted into the coefficients $F^{ \pm}$in Eq. (9) do not contribute to the current unless the correlators are anisotropic. Since we suppose the correlators to be isotropic, we neglect the integral terms in Eq. (14), and the nonideality of the interfaces just results in a renormalization of the transmission and reflection coefficients and the boundary conditions take the familiar form:

$$
f_{1 k \sigma}^{-}=R_{12 \sigma} f_{1 k \sigma}^{+}+T_{12 \sigma} f_{2 k \sigma}^{-}, \quad f_{2 k \sigma}^{+}=R_{21 \sigma} f_{2 k \sigma}^{-}+T_{21 \sigma} f_{1 k \sigma}^{+},
$$

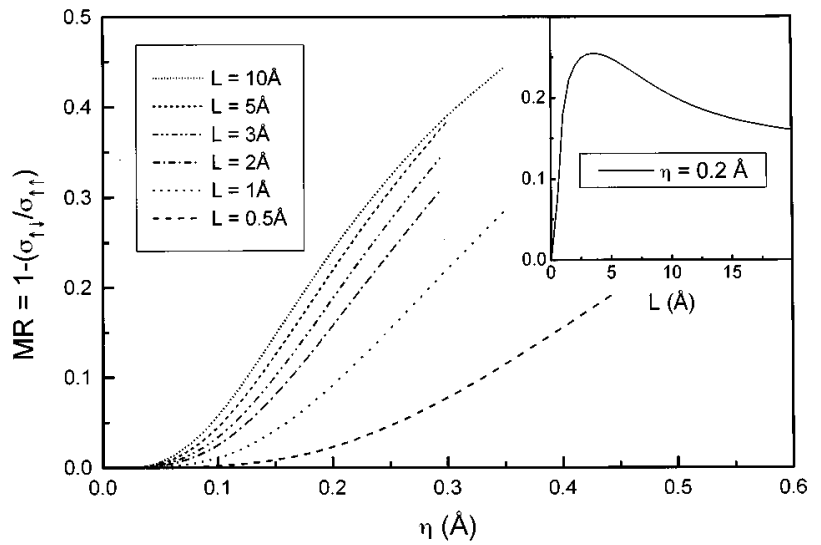

FIG. 2. MR effect for an $\mathrm{Fe}(20 \AA) / \mathrm{Cr}(10 \AA)$ superlattice calculated for different values of $\eta$ and $L$. The input parameters are $\varepsilon_{f, M}=8.23 \mathrm{eV}, \varepsilon_{f, m}=5.73 \mathrm{eV}, \varepsilon_{f, \mathrm{Cr}}=5.77 \mathrm{eV}$, all $\tau$ are taken equal $\left(5 \times 10^{-13} \mathrm{~s}\right), \lambda_{M}=6946 \AA, \lambda_{m}=5796 \AA$, and $\lambda_{\mathrm{Cr}}=5816 \AA$, and the effective electron mass $m$ is $1.5 \times m_{0}$. In the inset other parameters have been used, corresponding to an $\operatorname{Fe}(20 \AA) /$ $\mathrm{Cu}(10 \AA)$ superlattice. The input parameters used in the inset are $\varepsilon_{f, M}=8.23 \mathrm{eV}, \varepsilon_{f, m}=5.73 \mathrm{eV}, \varepsilon_{f, \mathrm{Cr}}=8.54 \mathrm{eV}$, all $\tau$ are taken equal $\left(5 \times 10^{-13} \mathrm{~s}\right), \lambda_{M}=6946 \AA, \lambda_{m}=5796 \AA$, and $\lambda_{\mathrm{Cu}}=7076 \AA$, the effective electron mass $m$ is $1.5 \times m_{0}$ and $\eta=0.2 \AA$. The MR effect is calculated as a function of $L$.

with $T$ and $R$ given by Eqs. (16), (17), and (18). For a perfectly flat interface $(\eta=0)$, these boundary conditions are determined only by $R^{0}$ and $T^{0}$, and are therefore equivalent to those obtained by Hood and Falicov. ${ }^{3}$ In this case the influence of the potential step at the interface due to different potentials in adjacent media is taken into account exactly.

From the boundary conditions Eq. (19) one can find the coefficients $F^{ \pm}$in the distribution functions of Eq. (5). For each configuration and spin direction we obtain six equations for $F_{i \sigma}^{ \pm}$, which can be reduced to a set of four equations with the help of two periodic boundary conditions at the outer surfaces given by Eq. (7). The solution of this set of equations being substituted into the conductivity of Eq. (9) gives us the integrands which depend on $R_{12}$ and $T_{12}$ from Eqs. (16)-(18).

We would like to add that ranges of integration in Eq. (9) include regions of full reflection. In these regions the $z$ component of the electron velocities in adjacent layers are equal to zero which corresponds to $R=1$ and $T=0$ and can result in electron channeling similar to the model of Hood and Falicov. $^{3}$

\section{NUMERICAL RESULTS}

After the calculation of the conductivities both for the $\mathrm{P}$ and AP configuration one can obtain the magnetoresistance from

$$
\mathrm{MR}=1-\frac{\sigma_{\uparrow \downarrow}}{\sigma_{\uparrow \uparrow}} .
$$

In Fig. 2 we calculated MR for a superlattice as a function of $\eta$, the deviation from the perfectly flat interface, for different values of the lateral in-plane correlation length $L$. The input parameters for the Fermi energy and relaxation times 


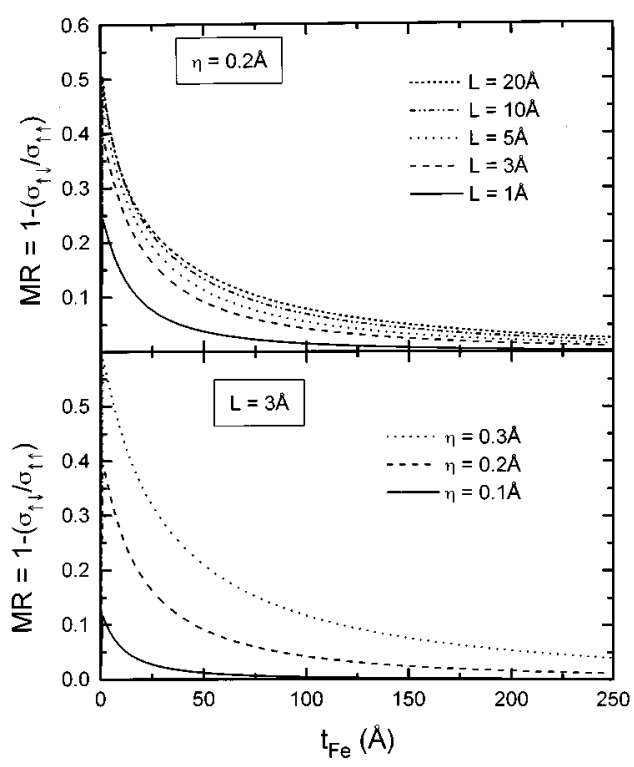

FIG. 3. MR effect for an $\mathrm{Fe}\left(t_{\mathrm{Fe}} \AA\right) / \mathrm{Cr}(10 \AA)$ superlattice calculated as a function of $t_{\mathrm{Fe}}$ for different values of $\eta$ and $L$. The input parameters are $\varepsilon_{f, M}=8.23 \mathrm{eV}, \varepsilon_{f, m}=5.73 \mathrm{eV}, \varepsilon_{f, \mathrm{Cr}}=5.77 \mathrm{eV}$, all $\tau$ are taken equal $\left(5 \times 10^{-13} \mathrm{~s}\right), \lambda_{M}=6946 \AA, \lambda_{M}=5796 \AA$, and $\lambda_{\mathrm{Cr}}=5816 \AA$, and the effective electron mass $m$ is $1.5 \times m_{0}$.

are taken from Hood and Falicov, ${ }^{3}$ with the values of the Fermi energies corresponding to $\mathrm{Fe}$ and $\mathrm{Cr}$. To concentrate on the effect of geometrical roughness only, all relaxation times are taken equal $\left(5 \times 10^{-13} \mathrm{~s}\right)$ such that there is no bulk spin-dependent scattering and furthermore no impurities at the interfaces were assumed. Note that the allowed values of $\gamma(\mathbf{q})$ and $\gamma^{\prime}(\mathbf{q})$ are restricted to small perturbations since our calculation of $R$ and $T$ [Eqs. (16), (17), and (18)] is based on a perturbation approach. This means, for instance, that the allowed values of $\eta$ should be small in comparison with the $z$ component of the electron wavelength. Therefore the maximum value of $\eta$ in our calculations amounts to $\eta \approx 0.45 \AA$. We will return to the value of this parameter in the discussion. In the inset of Fig. 2 we have used different parameters. We will come back to this point in the discussion.

Since we did not include any bulk spin-dependent scattering nor impurities at the interfaces in the parameters of Fig. 2 , we find that $M R=0$ when the interfaces are perfectly flat, like in the model of Hood and Falicov, which is identical to our model for perfectly flat interfaces. However, as is clear from Fig. 2, geometrical roughness $(\eta \neq 0$ and $L \neq 0)$ can already produce a MR effect without any other form of spindependent scattering. The MR effect here is due to the combination of geometrical roughness and different potentials for majority and minority electrons in the magnetic layers. Geometrical roughness will induce a larger probability of diffusive scattering for those electrons that experience the larger potential difference when crossing an interface. Thus for the parameters of Fig. 2 the majority electrons will be more diffusively scattered than the minority electrons in the case of geometrical roughness. This effect eventually results in different conductivities for parallel and antiparallel configurations of the magnetization.

In Figs. 3 and 4 the dependence of MR on the thickness of

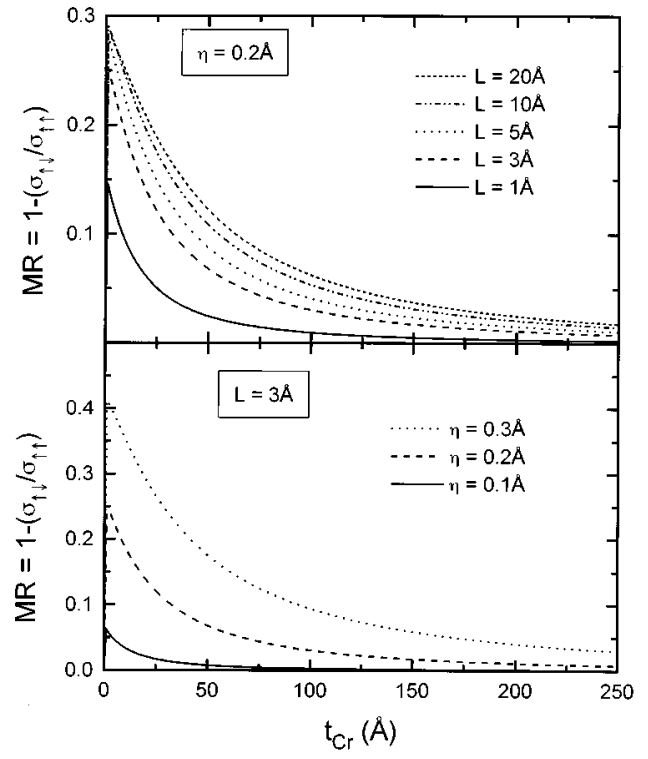

FIG. 4. MR effect for an $\mathrm{Fe}(20 \AA) / \mathrm{Cr}\left(t_{\mathrm{Cr}} \AA\right)$ superlattice calculated as a function of $t_{\mathrm{Cr}}$ for different values of $\eta$ and $L$. The input parameters are $\varepsilon_{f, M}=8.23 \mathrm{eV}, \varepsilon_{f, m}=5.73 \mathrm{eV}, \varepsilon_{f, \mathrm{Cr}}=5.77 \mathrm{eV}$, all $\tau$ are taken equal $\left(5 \times 10^{-13} \mathrm{~s}\right), \lambda_{M}=6946 \AA, \lambda_{m}=5796 \AA$, and $\lambda_{\mathrm{Cr}}=5816 \AA$, and the effective electron mass $m$ is $1.5 \times m_{0}$.

the $\mathrm{Fe}$ and $\mathrm{Cr}$ layers, respectively, is shown. Since there is no contribution to the spin-dependent scattering from the bulk of the layers, both figures display a monotonous decrease of MR as a function of layer thickness as one might expect when the MR effect is caused by geometrical interface roughness.

In Fig. 5 also a large bulk contribution to the spindependent scattering is taken into account by choosing different values for the relaxation times $\tau$ for majority and minoriy electrons. This results in a considerable MR effect even without interface roughness. In the bulk the minority electrons are most effectively scattered as a result of the parameters that we have chosen $\left(\tau_{m}<\tau_{M}\right)$. On the contrary, as stated above (Fig. 2), the interface roughness has more influence on the scattering of the majority electrons. Therefore for

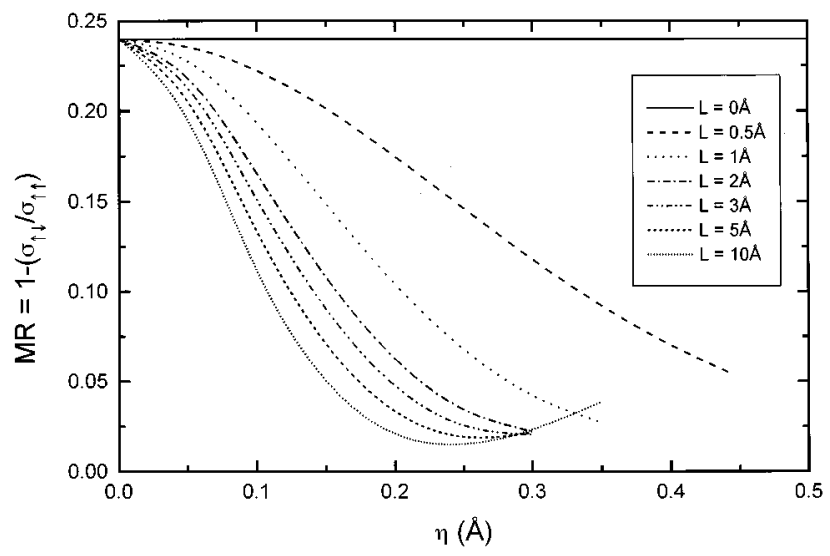

FIG. 5. MR effect for an $\operatorname{Fe}(20 \AA) / \mathrm{Cr}(10 \AA)$ superlattice calculated for different values of $\eta$ and $L$. The input parameters are $\varepsilon_{f, M}=8.23 \mathrm{eV}, \quad \varepsilon_{f, m}=5.73 \mathrm{eV}, \quad \varepsilon_{f, \mathrm{Cr}}=5.77 \mathrm{eV}, \quad \tau_{M}=5 \times 10^{-13}$, $\tau_{m}=0.5 \times 10^{-13}, \tau_{\mathrm{Cr}}=5 \times 10^{-13} \mathrm{~s}, \lambda_{M}=6946 \AA, \lambda_{m}=580 \AA$, and $\lambda_{\mathrm{Cr}}=5816 \AA$, and the effective electron mass $m$ is $1.5 \times m_{0}$. 


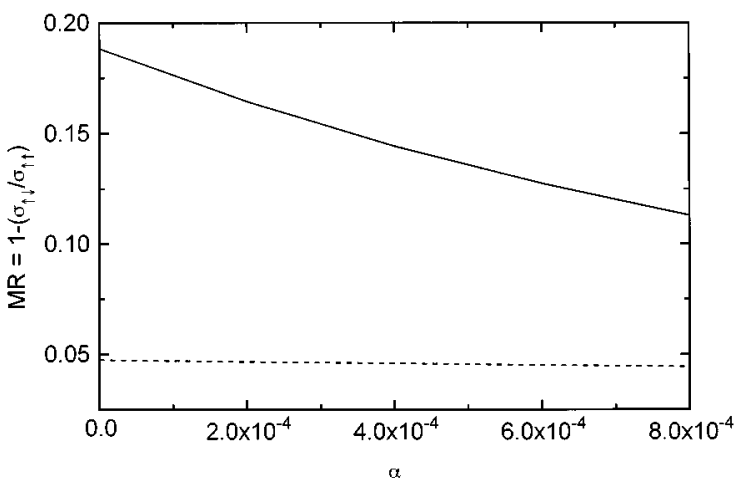

FIG. 6. MR effect for an $\operatorname{Fe}(20 \AA) / \mathrm{Cr}(10 \AA)$ superlattice calculated for different values of $\alpha$ (see text). The input parameters are $\eta=0.2 \AA, L=3 \AA, \varepsilon_{f, M}=8.23 \mathrm{eV}, \varepsilon_{f, m}=5.73 \mathrm{eV}, \varepsilon_{f, \mathrm{Cr}}=5.77 \mathrm{eV}$, all $\tau$ are taken equal $\left(5 \times 10^{-13} \mathrm{~s}\right), \lambda_{M}=6946 \AA, \lambda_{m}=5796 \AA$, and $\lambda_{\mathrm{Cr}}=5816 \AA$, and the effective electron mass $m$ is $1.5 \times m_{0}$.

the parameters of Fig. 5, an increase of the geometrical roughness decreases the scattering asymmetry between majority and minority electrons and therefore decreases the MR as well. For $L=10 \AA$ and $\eta \geqslant 0.25 \AA$, apparently the asymmetry due to roughness becomes dominant and MR increases again. Note that when we would have reversed the sign of the bulk contribution to the spin-dependent scattering (same potentials but $\tau_{M}=0.5 \times 10^{-13} \mathrm{~s}, \quad \lambda_{M}=695 \AA$, $\tau_{m}=5 \times 10^{-13} \mathrm{~s}, \lambda_{m}=5796 \AA$ ), the introduction of roughness indeed results in an increase of MR because now both bulk and interface scattering processes possess the same asymmetry.

Finally, in Fig. 6 the influence of impurities, defects, etc. at the interface is examined. Here the MR is calculated as a function of a parameter $\alpha$, which is given by

$$
\alpha=\frac{m^{2} \gamma^{\prime}(\mathbf{q})}{\hbar^{4}}
$$

As follows from Eqs. (17) and (18) also $\gamma^{\prime}$ will change the reflection and transmission coefficients for majority and minority electrons with a different amount. In Fig. 6 this results in a decrease of MR when there is no bulk spin-dependent scattering (solid line) as well as when bulk spin-dependent scattering is active (dashed line). In general however the effect of impurities, defects, etc. will depend on the specific choice of parameters such as potentials and relaxation times, and can also increase the MR.

\section{DISCUSSION}

As stated above the input parameters for the potential energy and relaxation times are taken from Hood and Falicov. ${ }^{3}$ It is known however that the choice of these parameters results in a conductivity that is too large in comparison with experiments which can be seen, e.g., by the unrealistic large values for the mean free paths. To compare their results with experiments Hood and Falicov therefore introduced a scaling factor to reduce the number of conduction electrons. As we do not intend to make a quantitative comparison with experimental data, we did not include a scaling factor in our calculations. We however would like to mention that when the relaxation times are reduced to obtain more realistic values of $\lambda$, this also reduces the MR effect with an order of magnitude.

Furthermore the Fermi velocity of the conduction electrons is in our model calculated on the basis of the freeelectron model. This is another reason why the values in our calculations should not be taken as exact numbers. Our results therefore merely represent trends in how the MR effect depends on the various input parameters and in particular on the geometrical roughness.

Let us now concentrate on the dependence of MR on $\eta$ and $L$ again. From Fig. 2 it is clear that geometrical roughness, which is in principle spin independent as we have chosen $\eta$ and $L$ to be spin independent, effectively leads to spin-dependent scattering which is induced by the different potentials for majority and minority electrons in the magnetic layers. The same result, although not shown in Fig. 2, holds for scattering at impurities and defects located at the interface and described by $\gamma^{\prime}$. As a result we cannot distinguish a spin-dependent interface mechanism from a spinindependent one.

According to our model, when there is no bulk spindependent scattering (all $\tau$ equal), there is only a MR effect when both $\eta \neq 0$ and $L \neq 0$. Also from Figs. 2, 3, and 4 we can see that an increase in $\eta$ or $L$ both seems to result in an increase of MR in the absence of bulk spin-dependent scattering. These results appear to be at variance with Hood and Falicov who find a significant MR already for zero correlation length $(L=0, \eta \neq 0)$. Moreover Barnas, ${ }^{8}$ who also describes interface roughness with the parameters $\eta$ and $L$ reports a decrease of MR with increasing $L$ which physically seems realistic as an infinite $L$ would correspond to a flat interface. This discrepancy can be understood however from the correlation function $\gamma(\mathbf{q})$, Eq. (4).

From Eq. (4) we can see that $\gamma=0$ when either $\eta=0$ or $L=0$, which explains the difference with Hood and Falicov. Equation (3) however has an uncertainty in the point $\rho=0$, $L=0$ and therefore is not suitable to describe fully uncorrelated roughness $(L=0)$. For this special case one should use a white-noise-type autocorrelation function ${ }^{11}\left[\right.$ like $\gamma^{\prime}(\boldsymbol{\rho})$ in Eq. (2) used for potential scattering]:

$$
\gamma(\boldsymbol{\rho})=\eta^{2} a^{2} \delta(\boldsymbol{\rho}),
$$

in which the value of $a$ is of the order of the lattice constant. When Eq. (22) is substituted into Eq. (18), our correction $\Delta R$ for the limiting case of an outer surface (which means $\left.v_{2}=0\right)$, is given by $\exp \left[-\left(c \eta^{2} \cos ^{2} \theta\right) / \lambda^{2}\right]$, expanded to the first order in $\eta^{2}$, where $c$ is a numerical constant, $\theta$ represents the angle of incidence, and $\lambda$ is the electron wavelength. The exponent mentioned above is the result obtained by Soffer ${ }^{12}$ for a surface with fully uncorrelated roughness. This means that we have extended the result of Soffer for the case of a rough interface.

Barnas ${ }^{8}$ has chosen a different correlation function to describe the interface roughness, viz.,

$$
\gamma(\mathbf{q})=2 \pi \eta^{2}\left(1+L^{2} q^{2}\right)^{-3 / 2} .
$$

This correlation function decreases monotonically as a function of $L$ and is thus maximal for $L=0$, in contrast with our correlation function [Eq. (4)] that shows a maximum as a function of $L$. Although we were not able to show this for the 
parameters in Fig. 2 because of the limitations imposed by the perturbation approach, the inset in Fig. 2, where we have chosen different parameters belonging to an $\mathrm{Fe} / \mathrm{Cu}$ multilayer, shows that this maximum in the correlation function results in a maximum in the MR effect. Thus for large values of $L$ our model behaves identically to the model of Barnas. $^{8}$

Another difference with the model of Barnas is that we do not imply that roughness scatters electrons in the same way as impurities do (i.e., by potential scattering), but obtain the boundary conditions from matching electron wave functions at the interfaces (i.e., a pure geometrical approach). As a result, we deduce the effective scattering amplitudes from our geometrical approach instead of introducing them from the beginning.

From an experimental point of view one cannot easily decide which approach is better. To start with, it is difficult to discern between interface spin-dependent scattering and bulk spin-dependent scattering. One of the difficulties is for instance that bulk and interface scattering cannot easily be isolated because a thick bulk layer will always be limited by interfaces, although a number of experimental studies have made it clear that the scattering at the interface between a magnetic and a nonmagnetic layer may be spin dependent. ${ }^{13,14}$ Furthermore, the role of interface roughness is still not clarified, mainly because it is difficult to vary the interface roughness in a controlled way and quantify it thereafter. Besides, changing, for instance, the growth conditions will surely influence the interfaces ${ }^{15}$ but might also affect the scattering processes in the bulk of the layers and hence complicate the interpretation of the measurements. It might therefore not be surprising that the conclusions drawn from a number of experiments seem to be contradictory. As an example, for the $\mathrm{Fe} / \mathrm{Cr}$ system a decreasing MR with increasing interface roughness is reported, ${ }^{16}$ as well as an increasing MR with increasing roughness, ${ }^{17}$ and the existence of an optimum roughness. ${ }^{18}$

Recently Beliën et al. ${ }^{19}$ have performed a detailed investigation of the relation between interface roughness and giant magnetoresistance in polycrystalline $\mathrm{Fe} / \mathrm{Cr}$ superlattices grown by molecular-beam epitaxy. In these superlattices the resistivity is mainly determined by interface roughness instead of bulk scattering. The interface quality was changed by varying the growth temperature between 0 and $400{ }^{\circ} \mathrm{C}$ and by the use of a $\mathrm{Cr}$ seed layer. It is concluded in this study that compositional mixing or interdiffusion decreases the magnetoresistance. It is however suggested that a small amount of steps at the interface can enhance the magnetoresistance which would be in agreement with our finding of an optimal value for the correlation length $L$.

\section{CONCLUSIONS}

We have solved the Boltzmann transport equation for magnetic superlattices with current-in-plane geometry. The boundary conditions for the electron distribution functions, needed to calculate the conductivity, were obtained by matching electron wave functions at the actual position of the rough interfaces. Geometrical roughness was described by two parameters, the root mean square of the deviations from the perfectly flat interface, $\eta$, and the in-plane correlation length, $L$. Other potential scattering agents at the interface were described by a random field $W(\boldsymbol{\rho})$. Although not intrinsically spin dependent, both kinds of interface nonideality $(\eta, L$ and $W)$ can result in effective spin-dependent scattering due to the spin-dependent potentials in the magnetic layers. It appears that all parameters can lead to an increase or decrease of the MR effect, depending on the values of the other parameters and the presence of bulk spindependent scattering.

\section{ACKNOWLEDGMENTS}

The authors would like to thank J. Vogels for his help in development of the software, C. H. W. Swüste for critical reading of the manuscript, and W. J. M. de Jonge for making this research possible and fruitful discussions. V.I.L. thanks Professor W. J. M. de Jonge for hospitality at the Eindhoven University of Technology.
${ }^{1}$ M. N. Baibich, J. M. Broto, A. Fert, F. Nguyen van Dau, F. Petroff, P. Etienne, G. Creuzet, A. Friederich, and J. Chaselas, Phys. Rev. Lett. 61, 2472 (1988); S. S. P. Parkin, ibid. 67, 3598 (1991); S. S. P. Parkin, N. More, and K. P. Roche, ibid. 64, 2304 (1990).

${ }^{2}$ R. E. Camley and J. Barnas, Phys. Rev. Lett. 63, 664 (1989); J. Barnas, A. Fuss, R. E. Camley, P. Grumberg, and W. Zinn, Phys. Rev. B 42, 8110 (1990); B. L. Johnson and R. E. Camley, ibid. 44, 9997 (1991).

${ }^{3}$ R. Q. Hood and L. M. Falicov, Phys. Rev. B 46, 8287 (1992); R. Q. Hood, L. M. Falicov, and D. R. Penn, ibid. 49, 368 (1994).

${ }^{4}$ P. M. Levy, S. Zhang, and A. Fert, Phys. Rev. Lett. 65, 1643 (1990); S. Zhang, P. M. Levy, and A. Fert, Phys. Rev. B 45, 8689 (1992).

${ }^{5}$ A. Vedyaev, C. Cowache, N. Ryzhanova, and B. Dieny, J. Phys. Condens. Matter 5, 8289 (1993); A. Vedyaev, B. Dieny, and N. Ryzhanova, Europhys. Lett. 19, 329 (1994).
${ }^{6}$ G. E. W. Bauer, Phys. Rev. Lett. 69, 1676 (1992); A. Brataas and G. E. W. Bauer, Europhys. Lett. 26, 117 (1994); P. B. Visscher, Phys. Rev. B 49, 3907 (1994).

${ }^{7}$ X. G. Zhang and W. H. Butler, Phys. Rev. B 51, 10085 (1995).

${ }^{8}$ J. Barnas and Y. Bruynseraede, Europhys. Lett. 32, 167 (1995); Phys. Rev. B 53, 5449 (1996).

${ }^{9}$ L. Falkovsky, Adv. Phys. 32, 753 (1983).

${ }^{10}$ V. K. Dugaev, V. I. Litvinov, and P. P. Petrov, Phys. Rev. B 52, 5306 (1995).

${ }^{11}$ G. Fishman and D. Calecki, Phys. Rev. Lett. 62, 1302 (1989).

${ }^{12}$ S. B. Soffer, J. Appl. Phys. 38, 1710 (1967).

${ }^{13}$ H. J. M. Swagten, G. J. Strijkers, G. L. J. Verschueren, M. M. H. Willekens, and W. J. M. de Jonge (unpublished).

${ }^{14}$ S. S. P. Parkin, Phys. Rev. Lett. 71, 1641 (1993).

${ }^{15}$ K. Takanashi, Y. Obi, Y. Mitani, and H. Fujimori, J. Phys. Soc. Jpn. 61, 1169 (1992); S. S. P. Parkin and B. R. York, Appl. Phys. Lett. 62, 1842 (1993); H. Kano, K. Kagawa, A. Suzuki, A. 
Okabe, K. Hayashi, and K. Aso, ibid. 63, 2839 (1993).

${ }^{16}$ N. M. Rensing, A. P. Payne, and B. M. Clemens, J. Magn. Magn. Mater. 121, 436 (1993).

${ }^{17}$ E. E. Fullerton, D. M. Kelly, J. Guimpel, I. K. Schuller, and Y. Bruynseraede, Phys. Rev. Lett. 68, 859 (1992).
${ }^{18}$ F. Petroff, A. Barthélémy, A. Hamzic, A. Fert, P. Etienne, S. Lequien, and G. Creuzet, J. Magn. Magn. Mater. 93, 95 (1991).

${ }^{19}$ P. Beliën, R. Schad, C. D. Potter, G. Verbanck, V. V. Moshchalkov, and Y. Bruynseraede, Phys. Rev. B 50, 9957 (1994). 